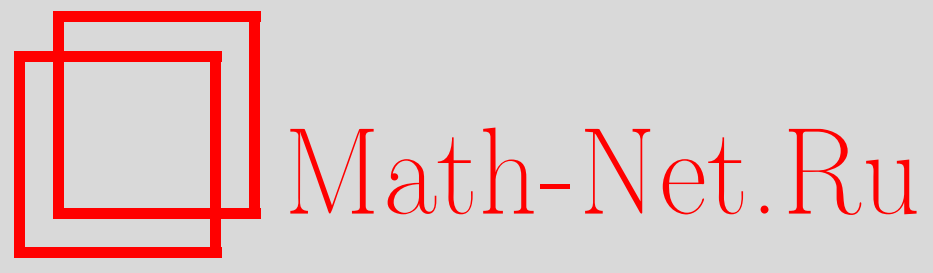

К. Б. Устинов, Д. С. Лисовенко, А. В. Ченцов, Ортотропная полоса с центральной полубесконечной трещиной под произвольными нормальными нагрузками, приложенными вдали от вершины трещины, Вестн. Сам. гос. техн. ун-та. Сер. Физ.мат. науки, 2019, номер 4, 657-670

DOI: https://doi.org/10.14498/vsgtu1736

Использование Общероссийского математического портала MathNet.Ru подразумевает, что вы прочитали и согласны с пользовательским соглашением

http://www. mathnet.ru/rus/agreement

Параметры загрузки:

IP : 54.166 .219 .16

26 апреля 2023 г., 06:50:42

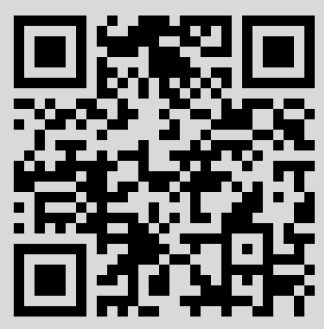


УДК 539.421

\title{
Ортотропная полоса с центральной полубесконечной трещиной под произвольными нормальными нагрузками, приложенными вдали от вершины трещины
}

\author{
К. Б. Устинов, Д. С. Лисовенко, А. В. Ченцов
}

Институт проблем механики им. А. Ю. Ишлинского РАН, Россия, 119526, Москва, просп. Вернадского, 101, корп. 1.

\section{Аннотация}

Проблемы распространения трещин в полосах привлекают внимание в основном из-за их важности для приложений: вычисление коэффициентов интенсивности напряжений для стандартных тестов, таких как трех- и четырехточечный изгиб; изучение разрушения в тонких слоистых структурах; изучение отслоения покрытий. Задача о нагружении полосы с центральной трещиной особенно интересна из-за ее относительной простоты, позволяющей анализировать и выделять существенные особенности процессов распространения трещин в структурах подобного типа. В работе получено точное аналитическое решение для задачи об ортотропной полосе с центральной полубесконечной трещиной, нормально нагруженной самоуравновешенной системой сил. Нагрузки приложены достаточно далеко от вершины трещины, что позволяет рассматривать нагрузку, как приложенную на бесконечности. Общее решение представлено как суперпозиция решений для двух случаев: симметрично приложенными моментами и поперечными силами с компенсирующими их моментами. Цель исследования состоит в нахождении коэффициентов при сингулярностях поля напряжений вблизи вершины трещины, то есть коэффициентов интенсивности напряжений. Решение задачи о раскрытии трещины получено для произвольного значения параметра анизотропии с помощью преобразований Лапласа для уравнений, связывающих усилия, действующие вдоль линии трещины, и производные относительных смещений берегов трещины. Коэффициент интенсивности напряжений для нагружения моментами совпадает

\section{Научная статья}

(2)(7) Контент публикуется на условиях лицензии Creative Commons Attribution 4.0 International (https://creativecommons.org/licenses/by/4.0/deed.ru)

\section{Образец для цитирования}

У стинов К. Б., Ли совенко Д. С., Ченцов А. В. Ортотропная полоса с центральной полубесконечной трещиной под произвольными нормальными нагрузками, приложенными вдали от вершины трещины // Вестн. Сам. гос. техн. ун-та. Сер. Физ.мат. науки, 2019. T. 23, № 4. C. 657-670. doi: 10.14498/vsgtu1736.

\section{Сведения об авторах}

Константин Борисович Устинов (1) https://orcid.org/0000-0001-5852-3355 доктор физико-математических наук, доцент; ведущий научный сотрудник; лаб. геомеханики; e-mail: ustinov@ipmnet.ru

Дмитрий Сергеевич Лисовенко (D) https://orcid.org/0000-0003-0485-3433

доктор физико-математических наук; заведующий лабораторией; лаб. механики технологических процессов; e-mail: lisovenk@ipmnet.ru

Александр Викторович Ченц,ов (1) https://orcid.org/0000-0003-4760-1738

кандидат физико-математических наук; старший научный сотрудник; лаб. механики технологических процессов; e-mail: chentsov@ipmnet.ru 
с элементарным решением теории пластин. Коэффициент интенсивности напряжений для нагружения силами получен в виде функции одного параметра, выраженного в виде однократного интеграла. Сравнение с имеющимися численными результатами продемонстрировало хорошее согласование решения в диапазоне параметра анизотропии, для которого были получены численные решения. Полученное решение охватывает все термодинамически допустимые значения параметров анизотропии.

Ключевые слова: коэффициент интенсивности напряжений, отслоение, интегральное преобразование, метод Винера-Хопфа.

Получение: 13 августа 2019 г. / Исправление: 4 ноября 2019 г. Принятие: 11 ноября 2019 г. / Публикация онлайн: 14 декабря 2019 г.

Введение. Проблемы распространения трещин в полосах привлекают внимание в основном из-за их важности для приложений: вычисление коэффициентов интенсивности напряжений (KИН) для стандартных тестов, таких как трех- и четырехточечный изгиб; изучение разрушения в тонких слоистых структурах; изучение отслоения покрытий. Задача о нагружении полосы с центральной трещиной особенно интересна из-за ее относительной простоты, позволяющей анализировать и выделять существенные особенности процессов распространения трещин в структурах подобного типа. В реальных ситуациях внешние нагрузки приложены на некоторых конечных расстояниях от вершины трещины. Однако если эти расстояния намного больше, чем толщина слоя, согласно принципу Сен-Венана их можно рассматривать как приложенные на бесконечности. Точность такого упрощения тем лучше, чем больше расстояние между точкой приложения нагрузки и вершиной трещины по сравнению с толщиной слоя.

Рассматриваемая задача исследовалась многими авторами как численно [1-7], так и аналитически [8]. Она является обобщением подобной задачи для изотропного слоя [9-12].

В частности, в работах $[9,10]$ показано, что произвольная нагрузка может быть разложена на четыре независимые моды, например: (i) моменты, симметрично приложенные к отделяющимся частям; (ii) продольные силы, приложенные в центральных точках отделяющихся частей, с компенсирующим моментом, приложенным к нижней части; (iii) поперечные силы с компенсирующими моментами, симметрично приложенные к отделяющимся частям; (iv) поперечные силы с компенсирующими моментами, приложенными к верхней отделяющейся части и к нерасслоившейся части. Такое разложение применимо как для изотропных, так и для анизотропных слоев, а также для составных слоев при различных упругих свойствах и толщинах слагающих слоев. Для рассматриваемой задачи существование симметрии позволяет выбирать четыре моды нагружения так, чтобы каждая из них вызывала бы либо раскрытие, либо сдвиг (для коэффициентов интенсивности напряжений $\mathrm{K}_{\mathrm{I}}$ и $\mathrm{K}_{\mathrm{II}}$ соответственно) [3]. В случае изотропии для каждой моды нагружения коэффициенты $\mathrm{K}_{\mathrm{I}}, \mathrm{K}_{\mathrm{II}}$ являются константами, причем две из них могут быть определены из элементарных решений, а две другие - численно [9] либо аналитически [12]. В случае кубической анизотропии коэффициенты $\mathrm{K}_{\mathrm{I}}$ и $\mathrm{K}_{\mathrm{II}}$ становятся функциями единственного параметра анизотропии - конкретной 
безразмерной комбинации упругих констант [3]. В случае ортотропии коэффициенты $\mathrm{K}_{\mathrm{I}}$ и $\mathrm{K}_{\mathrm{II}}$ становятся функциями двух параметров анизотропии: того же, что и для кубической анизотропии $\rho=\left(\beta_{66}+2 \beta_{12}\right) /\left(2 \sqrt{\beta_{11} \beta_{22}}\right)$, где $\beta_{i j}$ - коэффициенты податливости; $\lambda=\beta_{11} / \beta_{22}$. В [3] показано, что влияние последнего коэффициента может быть получено масштабированием результатов для кубической анизотропии, что выражается в появлении в выражениях для $\mathrm{K}_{\mathrm{I}}$ и $\mathrm{K}_{\mathrm{II}}$ степенных функций от параметра $\lambda$. Требование термодинамической устойчивости приводит к ограничениям $-1<\rho<\infty, 0<\lambda<\infty$; значение $\rho=1$ соответствует случаю изотропии и «вырожденной» анизотропии для ортотропных кристаллов; величина $\lambda=1$ - для кубических кристаллов и изотропных сред.

Точное аналитическое решение сформулированной задачи получено в [2] для случая нагружения сбалансированной парой сконцентрированных нормальных сил, приложенных на берегах трещины. С его помощью могут быть получены решения для двух рассматриваемых мод нагружения. Однако представленная форма решения делает затруднительным параметрический анализ.

В работе [3] численное решение получено для $0<\rho<4$ - диапазона, покрывающего большинство реальных кристаллов. Однако анализ параметров кристаллов выявил существование кристаллов с параметром $\rho$, выходящим далеко за пределы этого диапазона. На рис. 1 показана гистограмма распределения параметра $\rho$, вычисленного для плоского деформированного состояния реальных кристаллов кубической сингонии (данные получены на основе экспериментальных данных для коэффициентов податливости из [13]). В справочном издании Landolt-Börnstein [13] приводятся экспериментальные данные коэффициентов податливости для более чем 1100 кристаллов с кубической сингонией. В таблице представлены некоторые кристаллы кубической сингонии, для которых параметр $\rho$ сильно отличается от единицы.

В настоящей работе точное аналитическое решение задачи о раскрытии трещины получено для двух мод нагружения и произвольного значения параметра $\rho$ с помощью преобразований Лапласа для уравнений, связывающих усилия, действующие вдоль линии трещины, и производные относительных смещений берегов трещины.

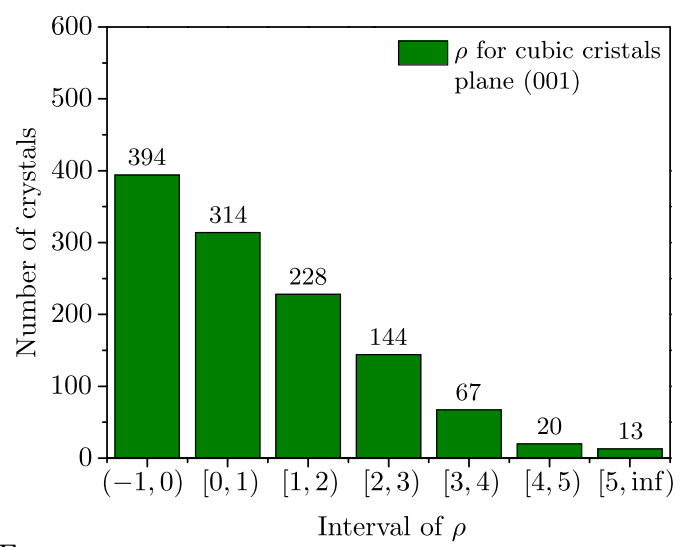

Рис. 1. Гистограммы распределения параметра $\rho$ для кристаллов кубической сингонии

[Figure 1. The histograms of parameter $\rho$ for cubic crystals] 
Значения параметра $\rho$ (для плоского деформированного состояния) для реальных кристаллов кубической сингонии [The values of parameter $\rho$ (for plane strain) for some real cubic crystals]

\begin{tabular}{|c|c||c|c|}
\hline Crystal & $\rho$ & Crystal & $\rho$ \\
\hline \hline $\operatorname{InTl}(27$ at $\% \mathrm{Tl})$ & -0.91 & $\mathrm{Li}$ & -0.63 \\
$\mathrm{CuZn}(45$ at $\% \mathrm{Zn})(\beta$-brass $)$ & -0.70 & $\mathrm{AlNi}(63.2$ at $\% \mathrm{Ni})$ & -0.63 \\
$\mathrm{CuAlNi}(14$ wt $\% \mathrm{Al}, 4.1$ wt $\% \mathrm{Ni})$ & -0.69 & $\mathrm{~K}$ & -0.57 \\
\hline
\end{tabular}

1. Постановка задачи. Рассмотрим упругую ортотропную полосу $-h<$ $<y<h$ с центральной полубесконечной трещиной $y=0, x<0$ (рис. 2). Соотношение между напряжениями и производными перемещения на границе полосы приведено в Приложении А. Предполагается, что удовлетворяются условия плоской деформации (или плоского напряженного состояния), механическое поведение определяется системой двумерных уравнений упругости (А.1)-(А.4). Границы $y= \pm h$ и $y=0, x<0$ предполагаются свободными от напряжений: $\sigma_{y y}=\sigma_{x y}=0$ для $y= \pm h$ и для $y=0, x<0$.

Предполагается, что нагрузка приложена на бесконечности в виде двух моментов $M$ поперечных сил $V$ (рис. 2). Для компенсации моментов, создаваемых поперечными силами, прикладываются дополнительные моменты $V l$ $(l \rightarrow \infty)$. Задача состоит в нахождении коэффициентов при сингулярностях поля напряжений вблизи вершины трещины, то есть коэффициентов интенсивности напряжений.

Из глобальных условий равновесия следует, что введенные параметры соответствуют интегральным силовым параметрам $[12,16]$

$$
M=-\int_{0}^{\infty} x \sigma_{y y}(x, 0) d x, \quad V=\int_{0}^{\infty} \sigma_{y y}(x, 0) d x
$$

В силу симметрии задачи касательные напряжения на продолжении трещины отсутствуют:

$$
\sigma_{x y}(x, 0)=0
$$

В точке изменения типа граничных условий (около нуля) напряжения

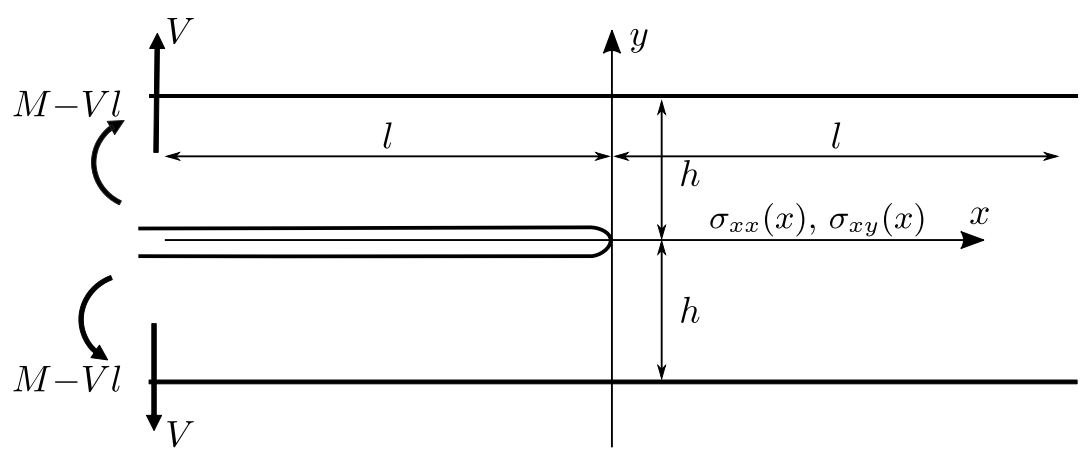

Рис. 2. Геометрия и система приложенных нагрузок

[Figure 2. The geometry and the applied loads] 
должны быть интегрируемыми:

$$
\sigma_{y y}=O\left(x^{-\nu}\right), \quad \nu<1
$$

Рассмотрим преобразования Лапласа (А.11) следующих величин:

$$
\begin{gathered}
F_{+}(p)=\int_{0}^{\infty} \sigma_{y y}(x, 0) e^{-p x} d x \\
F_{-}(p)=\int_{-\infty}^{0} \frac{\partial}{\partial x}\left(v^{+}(x, 0)-v^{-}(x, 0)\right) e^{-p x} d x .
\end{gathered}
$$

Здесь интервалы интегрирования были уменьшены за счет обращения в ноль напряжений для отрицательных аргументов и разрыва смещений для положительных аргументов. Равенство нулю подынтегральных выражений в (3), (4) для отрицательных и положительных аргументов следует из того факта, что $F_{+}(p), F_{-}(p)$ являются аналитическими функциями в правой $(\operatorname{Re} p>0)$ и левой $(\operatorname{Re} p<0)$ полуплоскостях комплексной переменной соответственно. Индексы «плюс» и «минус» в (4) соответствуют верхней и нижней граням трещины. Соотношения между производной смещений и напряжениями для верхней части задаются (А.16), аналогичные соотношения для нижней части можно получить из (А.16) заменив $h$ на $-h$. Таким образом, получаем скалярное уравнение для нормальной и сдвиговой компонент соответственно (контур $L$ соответствует мнимой оси и может быть преобразован в соответствии с правилами интегрирования контурных интегралов в комплексной плоскости):

$$
\begin{gathered}
F_{-}(p)=K(p) F_{+}(p), \quad p \in L, \\
K(p)=\frac{4 \beta_{11} \lambda^{-1} \sqrt{\rho^{2}-1}\left[\left(k_{1}+k_{2}\right) \sin \left(h p\left(k_{1}-k_{2}\right)\right)+\left(k_{1}-k_{2}\right) \sin \left(h p\left(k_{1}+k_{2}\right)\right)\right]}{-\left(k_{1}-k_{2}\right)^{2} \cos \left(h p\left(k_{1}+k_{2}\right)\right)+\left(k_{1}+k_{2}\right)^{2} \cos \left(h p\left(k_{1}-k_{2}\right)\right)-4 k_{1} k_{2}},
\end{gathered}
$$

где параметры $k_{1}$ и $k_{2}$ описаны в формуле (А.13).

Кроме того, должны выполняться условия в ключевых точках (ноль и бесконечность), вытекающие из (1), (2):

$$
\begin{array}{cl}
F_{+}(p)=N+M p+o(p), & \operatorname{Re} p \rightarrow 0+ \\
F_{+}(p)=O\left(p^{1-\nu}\right), \quad \nu<1, & \operatorname{Re} p \rightarrow+\infty .
\end{array}
$$

2. Решение задачи о раскрытии трещины. Ключевой этап решения (5) состоит в факторизации функции $K(p)$, т.е. ее представлении в виде произведения (отношения) двух функций, голоморфных в левой и правой полуплоскостях комплексной плоскости:

$$
K(p)=\Lambda_{-}^{-1}(p) \Lambda_{+}(p) .
$$

После нахождения $\Lambda_{ \pm}(p)$ решение получается с помощью теоремы Лиувилля [15]

$$
F_{ \pm}(p)=\Lambda_{ \pm}^{-1}(p) \Pi(p)
$$


Здесь П $(p)$ - функция с возможными полюсами и нулями в нуле и бесконечности, которая должна быть определена из условий (7), (8) в этих точках.

Используя (А.13), (6), уравнение (9) можно записать следующим образом:

$$
\begin{gathered}
\Lambda_{-}^{-1}(p) \Lambda_{+}(p)=-\frac{4 s 11}{\lambda^{3 / 4}} \sqrt{\frac{\rho+1}{2}} \operatorname{ctg}^{3}\left(p h \lambda^{-1 / 4}\right) G(p), \\
G(p)=\sqrt{\rho-1} \operatorname{tg}^{3}\left(p h \lambda^{-1 / 4}\right) \times \\
\times \frac{\sqrt{\rho+1} \sin \left(p h \lambda^{-1 / 4} \sqrt{2(\rho-1)}\right)+\sqrt{\rho-1} \sin \left(p h \lambda^{-1 / 4} \sqrt{2(\rho+1)}\right)}{(\rho-1) \cos \left(p h \lambda^{-1 / 4} \sqrt{2(\rho+1)}\right)-(\rho+1) \cos \left(p h \lambda^{-1 / 4} \sqrt{2(\rho-1)}\right)+2} .
\end{gathered}
$$

Здесь коэффициент при $G(p)$ в $(11)$ выбирается таким образом, чтобы функция $G(p)$ была голоморфна вдоль мнимой оси и приближалась к единице при стремлении $p$ к бесконечности вдоль мнимой оси.

Формально для $-1<\rho<1$ радикалы $\sqrt{\rho-1}$ в $(12)$ и других формулах становятся чисто мнимыми, однако они всегда появляются в мультипликативных парах, так что конечные выражения остаются действительными. Поэтому нет необходимости отдельно с самого начала рассматривать случай $-1<\rho<1$, поскольку представление части тригонометрических функций как гиперболических и наоборот приводит к одному и тому же конечному результату.

Используя представление $G(p)$ через интеграл Коши и стандартное представление котангенса в виде комбинации гамма-функций Эйлера $\Gamma(p)$ [15], факторизацию можно провести в следующей форме:

$$
\begin{gathered}
\Lambda_{+}(p)=\frac{\Gamma^{3}\left(1+p h \lambda^{-1 / 4} \pi^{-1}\right)}{\Gamma^{3}\left(1 / 2+p h \lambda^{-1 / 4} \pi^{-1}\right)} J_{+}(p), \\
\Lambda_{-}(p)=-\frac{\lambda^{3 / 4}}{4 s_{11}} \sqrt{\frac{2}{\rho+1} \frac{\Gamma^{3}\left(1 / 2-p h \lambda^{-1 / 4} \pi^{-1}\right)}{\Gamma^{3}\left(-p h \lambda^{-1 / 4} \pi^{-1}\right)} J_{-}(p),} \\
J_{ \pm}(p)=\exp \left\{\frac { 1 } { 2 \pi } \int _ { - \infty } ^ { \infty } \operatorname { l n } \left[\sqrt{\rho-1} \operatorname{th}^{3}\left(h s \lambda^{-1 / 4}\right) \times\right.\right. \\
\left.\left.\times \frac{\sqrt{\rho+1} \operatorname{sh}\left(h s \lambda^{-1 / 4} \sqrt{2(\rho-1)}\right)+\sqrt{\rho-1} \operatorname{sh}\left(h s \lambda^{-1 / 4} \sqrt{2(\rho+1)}\right)}{(\rho-1) \operatorname{ch}\left(h s \lambda^{-1 / 4} \sqrt{2(\rho+1)}\right)-(\rho+1) \operatorname{ch}\left(h s \lambda^{-1 / 4} \sqrt{2(\rho-1)}\right)+2}\right] \frac{d s}{i s-p}\right\}
\end{gathered}
$$

с асимптотиками

$$
\begin{aligned}
& \Lambda_{+}^{-1}(p)=\frac{\pi^{3 / 2}(\rho+1)^{1 / 4}}{2^{3 / 4} 3^{1 / 2}}\left[1-\frac{p h}{\lambda^{1 / 4}} Y_{1}(\rho)\right]+O\left(p^{2}\right) \\
& \Lambda_{+}^{-1}(p)=\left(\frac{p h}{\lambda^{1 / 4} \pi}\right)^{-3 / 2}+O\left(p^{-1 / 2}\right), \quad \operatorname{Re} p \rightarrow+\infty
\end{aligned}
$$

Здесь

$$
\begin{aligned}
& Y(\rho)=\frac{1}{\pi} \int_{-\infty}^{\infty} \frac{d L_{1}(s)}{d s} \frac{d s}{s}=\frac{1}{\pi} \int_{-\infty}^{\infty}[L(s)-L(0)] \frac{d s}{s^{2}} \\
& L(s)=\ln \left[s^{3} \sqrt{\rho-1} \frac{\sqrt{\rho+1} \operatorname{sh}(s \sqrt{2(\rho-1)})+\sqrt{\rho-1} \operatorname{sh}(s \sqrt{2(\rho+1)})}{(\rho-1) \operatorname{ch}(s \sqrt{2(\rho+1)})-(\rho+1) \operatorname{ch}(s \sqrt{2(\rho-1)})+2}\right] .
\end{aligned}
$$


Для $-1<\rho<1$ эта формула может быть переписана так:

$$
L(s)=\ln \left[s^{3} \sqrt{1-\rho} \frac{\sqrt{\rho+1} \sin (s \sqrt{2(1-\rho)})+\sqrt{1-\rho} \operatorname{sh}(s \sqrt{2(\rho+1)})}{(\rho-1) \operatorname{ch}(s \sqrt{2(\rho+1)})-(\rho+1) \cos (s \sqrt{2(1-\rho)})+2}\right] .
$$

Анализ (10), (16), (17) совместно с (7), (8) приводит к представлению

$$
\Pi(p)=\frac{2^{3 / 4} 3^{1 / 2}}{\pi^{3 / 2}(\rho+1)^{1 / 4}}\left[N+\left(M+\frac{h}{\lambda^{1 / 4}} Y(\rho) N\right) p\right] .
$$

Появление в (18) членов с отрицательной степенью $p$ нарушило бы условие (8), в то время как появление членов со степенью $p$, большей единицы, нарушило бы условие (7).

Таким образом, (10), (13), (14), (15), (18) дают решение задачи.

Рассмотрим асимптотику для $p \rightarrow+\infty$. Подстановка (17), (18) в (10) приводит к соотношению

$$
F_{+}(p)=6^{1 / 2} \lambda^{3 / 8}\left(\frac{2}{\rho+1}\right)^{1 / 4}\left(M+\frac{h}{\lambda^{1 / 4}} Y_{1}(\rho) N\right) h^{-3 / 2} p^{-1 / 2}+o\left(p^{-1 / 2}\right)
$$

$\operatorname{Re} p \rightarrow+\infty$.

Использование теоремы Абеля [15] дает асимптотику напряжений для $x \rightarrow 0+:$

$$
\sigma_{y y}=6^{1 / 2} \pi^{-1 / 2} \lambda^{3 / 8}\left(\frac{2}{\rho+1}\right)^{1 / 4}\left(M+\frac{h}{\lambda^{1 / 4}} Y(\rho) N\right) h^{-3 / 2} x^{-1 / 2}+o\left(x^{-1 / 2}\right) .
$$

Коэффициент интенсивности напряжений тогда выражается так:

$$
K_{\mathrm{I}} h^{3 / 2}=12^{1 / 2} \lambda^{3 / 8}\left(\frac{2}{\rho+1}\right)^{1 / 4}\left(M+\lambda^{-1 / 4} Y(\rho) N h\right) \text {. }
$$

Форма (19) совпадает с формой из [1,3], где результаты были получены с использованием масштабирования [2], а функции $Y(\rho)$ были рассчитаны методом конечных элементов.

\section{3. Численные результаты и сравнение с предыдущими исследо-} ваниями. Коэффициент интенсивности напряжений (19) при $V=0$ совпадает с элементарным решением, полученным при рассмотрении энергий изгиба и растяжения ортотропных балок и нахождении коэффициентов интенсивности напряжений с использованием их соотношений с выделением энергии [17].

Решение для $M=0, V \neq 0$ хорошо согласуется с решением [3]

$$
Y(\rho)=0.677+0.146(\rho-1)-0.0178(\rho-1)^{2}+0.00242(\rho-1)^{3},
$$

полученным путем интерполяции результатов MKЭ (рис. 3). Отметим, что формулы (20) были получены для области «типичных значений» $0 \leqslant \rho \leqslant 4$ [3] и они не обязаны давать приемлемые результаты вне этой области. Значения $Y(\rho)$, а также численное решение (20) [3] представлены на рис. 3. 


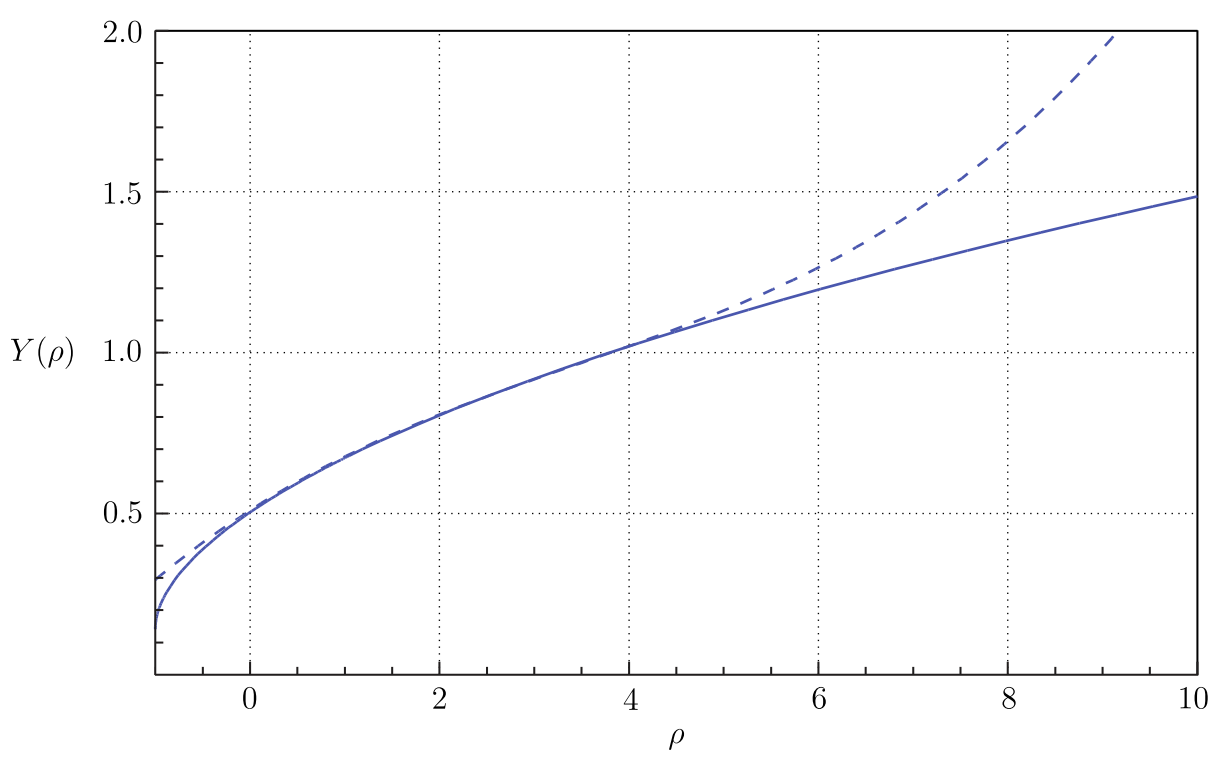

Рис. 3. Зависимость $Y(\rho)$; сплошные линии соответствуют полученному решению, пунктирные линии соответствуют решению из [3]

[Figure 3. The $Y(\rho)$ compared. Solid line correspond to the obtained solution, dashed line is according to the solution from [3]]

Функция $Y(\rho)$ близка к квадратно-корневым зависимостям, по крайней мере, для экстремальных значений $\rho$. Для промежуточных значений $0 \leqslant \rho \leqslant 4$ зависимости из [3] с достаточной точностью совпадают с полученным решением.

Выводы. Для задачи об ортотропной полосе с центральной полубесконечной трещиной, нагруженной самоуравновешенной системой нормальных усилий, получено точное аналитическое решение. Нагрузка приложена достаточно далеко от вершины трещины, чтобы рассматривать ее как приложенную на бесконечности. Общее решение выражается в виде суперпозиции решений для двух типов нагружения, соответствующих паре симметрично приложенных моментов и паре сил с компенсирующими моментами. Коэффициент интенсивности напряжений для первого случая совпадает с элементарным решением теории пластин. Коэффициент интенсивности напряжений для второго случая получен в виде двух функций одного параметра, выраженного в виде однократного интеграла. Сравнение с имеющимися численными результатами демонстрирует хорошее согласование решения в диапазоне параметра анизотропии, для которого были получены численные решения. Полученное решение охватывает все термодинамически допустимые значения параметров анизотропии. 
Приложение. Соотношения между напряжениями и производными перемещения на границе полосы. Система двумерных уравнений упругости для ортотропных сред в декартовых координатах $x y$, совпадающих с главными осями напряжений, может быть написана следующим образом:

1. Уравнения равновесия

$$
\frac{\partial \sigma_{x x}}{\partial x}+\frac{\partial \sigma_{x y}}{\partial y}=0, \quad \frac{\partial \sigma_{x y}}{\partial x}+\frac{\partial \sigma_{y y}}{\partial y}=0 .
$$

Здесь $\sigma_{x x}, \sigma_{y y}, \sigma_{x y}$ - компоненты тензора напряжений.

2. Соотношения Коши между деформациями $\varepsilon_{x x}, \varepsilon_{y y}, \varepsilon_{x y}$ и перемещениями $u, v$ :

$$
\varepsilon_{x x}=\frac{\partial u}{\partial x}, \quad \varepsilon_{y y}=\frac{\partial v}{\partial y}, \quad \varepsilon_{x y}=\frac{1}{2}\left(\frac{\partial u}{\partial y}+\frac{\partial v}{\partial x}\right) .
$$

3. Уравнение совместности, связывающее компоненты тензора деформации, непосредственно следующее из (А.2):

$$
\frac{\partial^{2}}{\partial x^{2}} \varepsilon_{y y}+\frac{\partial^{2}}{\partial y^{2}} \varepsilon_{x x}=2 \frac{\partial^{2}}{\partial x \partial y} \varepsilon_{x y} .
$$

4. Закон Гука для кристаллов с кубической анизотропией для плоского напряженного состояния:

$$
\begin{aligned}
& \varepsilon_{x x}=s_{11} \sigma_{x x}+s_{12} \sigma_{y y}, \\
& \varepsilon_{y y}=s_{12} \sigma_{x x}+s_{22} \sigma_{y y}, \\
& 2 \varepsilon_{x y}=s_{66} \sigma_{x y} .
\end{aligned}
$$

Здесь $s_{j k}$ - коэффициенты податливости. Для плоского деформированного состояния коэффициенты податливости $s_{j k}$ должны быть заменены модифицированными коэффициентами $\beta_{j k}$ :

$$
\beta_{j k}=s_{j k}-\frac{s_{j 3} s_{k 3}}{s_{33}} .
$$

Введение функции напряжений Эйри $F$ в виде

$$
\sigma_{x x}=\frac{\partial^{2} F}{\partial y^{2}}, \quad \sigma_{y y}=\frac{\partial^{2} F}{\partial x^{2}}, \quad \sigma_{x y}=-\frac{\partial^{2} F}{\partial x \partial y}
$$

позволяет автоматически удовлетворить уравнениям равновесия (А.1). Замена (A.5) в (А.4) и затем в (А.3) сокращает систему уравнений упругости для плоской деформации до одного уравнения относительно одного неизвестного (функция напряжения) [14]:

$$
\beta_{22} \frac{\partial^{4} F}{\partial x^{4}}+\left(\beta_{66}+2 \beta_{12}\right) \frac{\partial^{4} F}{\partial x^{2} \partial y^{2}}+\beta_{11} \frac{\partial^{4} F}{\partial y^{4}}=0
$$

Из (А.5), (А.6) следует, и на этот факт указывают много исследований, например [14], что для предписанной геометрии и граничных условий (заданных в терминах напряжений) поле напряжений определяется двумя безразмерными комбинациями упругих констант. В качестве этих комбинаций мы 
выбрали используемые в работе [2] величины:

$$
\lambda=\frac{\beta_{11}}{\beta_{22}}, \quad \rho=\frac{\beta_{66}+2 \beta_{12}}{2 \sqrt{\beta_{11} \beta_{22}}} .
$$

Ограничения для этих величин накладываются положительной определенностью упругой энергии

$$
0<\lambda<\infty, \quad-1<\rho<\infty
$$

Тогда уравнение (А.6) представляется в виде

$$
\frac{\partial^{4} F}{\partial x^{4}}+2 \sqrt{\lambda} \rho \frac{\partial^{4} F}{\partial x^{2} \partial y^{2}}+\lambda \frac{\partial^{4} F}{\partial y^{4}}=0 .
$$

Рассмотрим вспомогательную двумерную задачу об ортотропной упругой полосе $0 \leqslant y \leqslant h$ с верхней границей, свободной от напряжений:

$$
\sigma_{y y}(x, h)=\sigma_{x y}(x, h)=0,
$$

и нижней границей - под действием поля напряжений:

$$
\sigma_{x y}(x, 0)=0, \quad \sigma_{y y}(x, 0)=q_{y}(x) .
$$

Здесь $q_{y}(x)$ - известная функция.

Найдем соотношение между напряжениями, действующими вдоль нижней границы и производной компонент смещений $\frac{\partial v}{\partial x}(x, 0)$, которая выражается в терминах функции напряжений $F$ с использованием (А.5), (А.2), (А.4) следующим образом:

$$
v^{\prime}=\frac{\partial v}{\partial x}=-\left(\beta_{66}+\beta_{12}\right) \frac{\partial^{2} F}{\partial x \partial y}-\beta_{11} \int \frac{\partial^{3} F}{\partial y^{3}} d x .
$$

Общее решение (А.6) для бесконечной полосы может быть получено с помощью двустороннего преобразования Лапласа:

$$
\hat{f}(p, y)=\int_{-\infty}^{\infty} f(x, y) e^{-p x} d x
$$

и обратного преобразования:

$$
f(x, y)=-\frac{1}{2 \pi i} \int_{L} \hat{f}(p, y) e^{p x} d p
$$

где контур $L$ соответствует мнимой оси, а направление интегрирования сверху вниз.

Применение (А.11) к (А.7) приводит к уравнению

$$
p^{4} \hat{F}(p, y)+2 \sqrt{\lambda} \rho p^{2} \frac{\partial^{2} \hat{F}(p, y)}{\partial y^{2}}+\lambda \frac{\partial^{4} \hat{F}(p, y)}{\partial y^{4}}=0
$$


Общее решение (А.12) запишется так:

$$
\hat{F}(p, y)=C_{1} \cos \left(k_{1} p y\right)+C_{2} \cos \left(k_{2} p y\right)+C_{3} \sin \left(k_{1} p y\right)+C_{4} \sin \left(k_{2} p y\right),
$$

где

$$
\begin{gathered}
k_{1,2}=\lambda^{-1 / 4} \sqrt{\rho \pm \sqrt{\rho^{2}-1}}, \quad k_{1} \pm k_{2}=\lambda^{-1 / 4} \sqrt{2} \sqrt{\rho \pm 1} \\
k_{1} k_{2}=\lambda^{-1 / 2}, \quad k_{1}^{2}+k_{2}^{2}=2 \lambda^{-1 / 2} \rho, \quad k_{1}^{2}-k_{2}^{2}=2 \lambda^{-1 / 2} \sqrt{\rho^{2}-1} .
\end{gathered}
$$

Применение (А.11) к (А.5), (А.10) дает соотношения для преобразования для следующих величин:

$$
\begin{gathered}
\hat{\sigma}_{x x}=\frac{\partial^{2} \hat{F}}{\partial y^{2}}, \quad \hat{\sigma}_{y y}=p^{2} \hat{F}, \quad \hat{\sigma}_{x y}=-p \frac{\partial \hat{F}}{\partial y} \\
\hat{v}^{\prime}=-\left(\beta_{66}+\beta_{12}\right) p \frac{\partial \hat{F}}{\partial y}-\frac{1}{p} \beta_{11} \frac{\partial^{3} \hat{F}}{\partial y^{3}} .
\end{gathered}
$$

Подстановка последних двух формул (А.14) в преобразования (А.8), (А.9):

$$
\begin{gathered}
\hat{\sigma}_{x y}(p, 1)=\hat{\sigma}_{y y}(p, 1)=0, \\
\hat{\sigma}_{x y}(p, 0)=0, \quad \hat{\sigma}_{y y}(p, 0)=\hat{q}_{y}(p)
\end{gathered}
$$

позволяет выразить константы $C_{\mathrm{I}}$ через $\hat{q}_{y}$, подстановка которых в (А.15) приводит к искомому соотношению:

$$
\hat{v}^{\prime}=\frac{\beta_{22}\left(k_{1}^{2}-k_{2}^{2}\right)\left(k_{1} \cos \left(h k_{2} p\right) \sin \left(h k_{1} p\right)-k_{2} \sin \left(h k_{2} p\right) \cos \left(h k_{1} p\right)\right)}{k_{1} k_{2}\left(\left(k_{1}^{2}+k_{2}^{2}\right) \sin \left(h k_{1} p\right) \sin \left(h k_{2} p\right)+2 k_{1} k_{2}\left(\cos \left(h k_{1} p\right) \cos \left(h k_{2} p\right)-1\right)\right)} \hat{q}_{y}(p) .
$$

Конкурирующие интересы. Мы заявляем, что у нас нет конфликта интересов в авторстве и публикации этой статьи.

Авторский вклад и ответственность. Все авторы принимали участие в разработке концепции статьи и в написании рукописи. Авторы несут полную ответственность за предоставление окончательной рукописи в печать. Окончательная версия рукописи была одобрена всеми авторами.

Финансирование. Работа выполнена при поддержке Российского Научного Фонда (проект № 18-79-10270).

\section{Библиографический список}

1. Suo Z., Bao G., Fan B., Wang T. C. Orthotropy rescaling and implications for fracture in composites// Int. J. Solids Struct., 1991. vol.28, no.2. pp. 235-248. doi:10.1016/ 0020-7683 (91) 90208-W.

2. Suo Z. Domination specimens for orthotopic materials // J. Appl. Mech. - T. ASME, 1990. vol. 57, no. 3. pp. 627-634. doi : 10.1115/1.2897068.

3. Bao G., Ho S., Suo Z., Fan B. The role of material orthotropy in fracture specimens for composites // Int. J. Solids Struct., 1992. vol.29, no.9. pp. 1105-1116. doi: 10.1016/ 0020-7683(92) 90138- J. 
4. Li S., Wang J., Thouless M. D. The effects of shear on delamination in layered materials // J. Mech. Phys. Sol., 2004. vol.52, no.1. pp. 193-214. doi: 10.1016/S0022-5096(03) 00070-X.

5. Massabo R., Brandinelli L., Cox B. N. Mode I weight functions for an orthotropic double cantilever beam // Int. J. Eng. Sci., 2003. vol.41, no.13-14. pp. 1497-1518. doi: 10.1016/ S0020-7225 (03) 00029-6.

6. Brandinelli L., Massabo R. Mode II weight functions for isotropic and orthotropic double cantilever beams// Int. J. Fract., 2006. vol.139, no.1. pp. 1-25. doi:10.1007/ s10704-006-6358-0.

7. Thouless M. D. Phase angles and delamination of layered materials // Eng. Fract. Mech., 2018. vol. 191. pp. 153-167. doi: 10.1016/j.engfracmech.2018.01.033.

8. Georgiadis H. G., Papadopoulos G. A. Elastostatics of the orthotropic double-cantileverbeam fracture specimen // Z. angew. Math. Phys., 1990. vol. 41, no. 6. pp. 889-899. doi: 10. $1007 / B F 00945841$.

9. Suo Z., Hutchinson J. W. Interface crack between two elastic layers // Int. J. Fract., 1990. vol. 43, no. 1. pp. 1-18. doi: 10.1007/BF00018123.

10. Hutchinson J. W., Suo Z. Mixed mode cracking in layered materials // Adv. Appl. Mech., 1991. vol. 29. pp. 63-191. doi : 10.1016/S0065-2156(08) 70164-9.

11. Begley M. R., Hutchinson J. W. The Mechanics and Reliability of Films, Multilayers and Coatings. Cambridge, United Kingdom: Cambridge Univ. Press, 2017. x+278 pp. doi: 10 . 1017/9781316443606.

12. Ustinov K. On semi-infinite interface crack in bi-material elastic layer // Eur. J. Mech. A-Solid., 2019. vol.75. pp. 56-69. doi: 10.1016/j.euromechsol.2019.01.013.

13. Low Frequency Properties of Dielectric Crystals. Second and Higher Order Elastic Constants / Landolt-Börnstein - Group III Condensed Matter. vol.29A/ ed. D. F. Nelson, 1992. doi : $10.1007 / \mathrm{b} 44185$.

14. Лехницкий С. Г. Теория упругости анизотропного тела. М.: Наука, 1977. 416 с.

15. Noble B. Methods Based on the Wiener-Hopf Technique for the Solution of Partial Differential Equations. New York: Chelsea Publ., 1988. x+246 pp.

16. Устинов К. Б. Об отслоении слоя от полуплоскости; условия упругой заделки для пластины, эквивалентной слою// Изв. РАН. MTT, 2015. № 1. С. 75-95.

17. Sih G. C.,Paris P. C.,Irwin G. R. On cracks in rectilinearly anisotropic bodies // Int. J. Fract. Mech., 1965. vol. 1, no. 3. pp. 189-203. doi: 10.1007/BF00186854. 


\title{
Orthotropic strip with central semi-infinite crack under arbitrary loads applied far apart from the crack tip
}

\author{
K. B. Ustinov, D. S. Lisovenko, A. V. Chentsov \\ A. Ishlinsky Institite for Problems in Mechanics, Russian Academy of Sciences, \\ 101, pr. Vernadskogo, Moscow, 119526, Russian Federation.
}

\begin{abstract}
The exact analytical solution has been obtained for a problem of orthotropic strip with central semi-infinite crack loaded normally with selfbalanced system of forces applied far enough from the crack tip to be considered as applied at infinity. The general solution is expressed as a superposition of solutions for two modes of loading: (i) symmetrically applied moments; (ii) symmetrically applied transverse forces with compensating moments. The exact expressions for stress intensity factor (SIF) have been obtained. Due to symmetry only the opening mode of SIF is present for each case of loading. For both cases of loading the stress states are determined by two dimensionless parameters composed by four elastic constants. Expression for SIF for the case of loading with symmetrically applied moments is obtained in terms of elementary functions and coincides with the elementary solution due to beam theory. Expression for SIF for the case of loading with symmetrically applied transverse forces with compensating moments has been obtained in terms of one function of one of the parameters expressed as a single integral, multiplied by a power function of the second parameter. The solution for this case demonstrated good agreement with the existing numerical solution for the range of parameters, for which the latter had been obtained. The obtained solution covers all possible range of parameters.
\end{abstract}

Keywords: stress intensity factor, delamination, integral transform, WienerHopf technique.

Received: $13^{\text {th }}$ August, 2019 / Revised: $4^{\text {th }}$ November, $2019 /$

Accepted: $11^{\text {th }}$ November, $2019 /$ First online: $14^{\text {th }}$ December, 2019

\begin{abstract}
Research Article
(2) (a) The content is published under the terms of the Creative Commons Attribution 4.0 International License (http://creativecommons.org/licenses/by/4.0/)

Please cite this article in press as:

Ustinov K. B., Lisovenko D. S., Chentsov A. V. Orthotropic strip with central semiinfinite crack under arbitrary loads applied far apart from the crack tip, Vestn. Samar. Gos. Tekhn. Univ., Ser. Fiz.-Mat. Nauki [J. Samara State Tech. Univ., Ser. Phys. Math. Sci.], 2019, vol. 23, no. 4, pp. 657-670. doi: 10.14498/vsgtu1736 (In Russian).
\end{abstract}

\section{Authors' Details:}

Konstantin B. Ustinov (10) https://orcid.org/0000-0001-5852-3355

Dr. Phys. \& Math. Sci., Associate Professor; Leading Researcher; Lab. of Geomechanics; e-mail: ustinov@ipmnet.ru

Dmitry S. Lisovenko (D) https://orcid.org/0000-0003-0485-3433

Dr. Phys. \& Math. Sci.; Head of Lab.; Lab. of Mechanics of Technological Processes;

e-mail: lisovenk@ipmnet.ru

Alexander V. Chentsov (1) https://orcid.org/0000-0003-4760-1738

Cand. Phys. \& Math. Sci., Associate Professor; Senior Researcher; Lab. of Mechanics of Technological Processes; e-mail: chentsov@ipmnet.ru 
Competing interests. We declare that we have no competing interests.

Authors' contributions and responsibilities. Each author has participated in the article concept development and in the manuscript writing. The authors are absolutely responsible for submitting the final manuscript in print. Each author has approved the final version of manuscript.

Funding. This work was supported by the Russian Science Foundation (project no. 1879-10270).

\section{References}

1. Suo Z., Bao G., Fan B., Wang T. C. Orthotropy rescaling and implications for fracture in composites, Int. J. Solids Struct., 1991, vol.28, no. 2, pp. 235-248. doi: 10.1016/ 0020-7683 (91) 90208-W.

2. Suo Z. Domination specimens for orthotopic materials, J. Appl. Mech. - T. ASME, 1990, vol. 57, no. 3, pp. 627-634. doi : 10.1115/1.2897068.

3. Bao G., Ho S., Suo Z., Fan B. The role of material orthotropy in fracture specimens for composites, Int. J. Solids Struct., 1992, vol.29, no.9, pp. 1105-1116. doi: 10.1016/ 0020-7683(92) 90138-J.

4. Li S., Wang J., Thouless M. D. The effects of shear on delamination in layered materials, J. Mech. Phys. Sol., 2004, vol.52, no.1, pp. 193-214. doi:10.1016/S0022-5096(03) 00070-X.

5. Massabo R., Brandinelli L., Cox B. N. Mode I weight functions for an orthotropic double cantilever beam, Int. J. Eng. Sci., 2003, vol.41, no.13-14, pp. 1497-1518. doi: 10.1016/ S0020-7225 (03) 00029-6.

6. Brandinelli L., Massabo R. Mode II weight functions for isotropic and orthotropic double cantilever beams, Int. J. Fract., 2006, vol.139, no.1, pp. 1-25. doi:10.1007/ s10704-006-6358-0.

7. Thouless M. D. Phase angles and delamination of layered materials, Eng. Fract. Mech., 2018, vol. 191, pp. 153-167. doi: 10.1016/j.engfracmech.2018.01.033.

8. Georgiadis H. G., Papadopoulos G. A. Elastostatics of the orthotropic double-cantileverbeam fracture specimen, Z. angew. Math. Phys., 1990, vol. 41, no.6, pp. 889-899. doi: 10. $1007 / B F 00945841$.

9. Suo Z., Hutchinson J. W. Interface crack between two elastic layers, Int. J. Fract., 1990, vol. 43, no. 1, pp. 1-18. doi : 10.1007/BF00018123.

10. Hutchinson J. W., Suo Z. Mixed mode cracking in layered materials, Adv. Appl. Mech., 1991, vol. 29, pp. 63-191. doi: 10.1016/S0065-2156(08)70164-9.

11. Begley M. R., Hutchinson J. W. The Mechanics and Reliability of Films, Multilayers and Coatings. Cambridge, United Kingdom, Cambridge Univ. Press, 2017, x+278 pp. doi: 10 . $1017 / 9781316443606$.

12. Ustinov K. On semi-infinite interface crack in bi-material elastic layer, Eur. J. Mech. ASolid., 2019, vol. 75, pp. 56-69. doi: 10.1016/j.euromechsol.2019.01.013.

13. Low Frequency Properties of Dielectric Crystals. Second and Higher Order Elastic Constants, Landolt-Börnstein — Group III Condensed Matter, vol. 29A, ed. D. F. Nelson, 1992. doi: $10.1007 / \mathrm{b} 44185$.

14. Lekhnitsky S. G. Theory of Elasticity of an Anisotropic Elastic Body. Mosocw, Mir, 1981, 430 pp.

15. Noble B. Methods Based on the Wiener-Hopf Technique for the Solution of Partial Differential Equations. New York, Chelsea Publ., 1988, x+246 pp.

16. Ustinov K. B. On separation of a layer from the half-plane: Elastic fixation conditions for a plate equivalent to the layer, Mech. Solids, 2015, vol.50, no. 1, pp. 62-80. doi: 10.3103/ S0025654415010070.

17. Sih G. C.,Paris P. C.,Irwin G. R. On cracks in rectilinearly anisotropic bodies, Int. J. Fract. Mech., 1965, vol. 1, no.3, pp. 189-203. doi:10.1007/BF00186854. 